\title{
ON COUPLINGS OF SYMMETRIC OPERATORS WITH POSSIBLY UNEQUAL AND INFINITE DEFICIENCY INDICES
}

\author{
V. I. MogILEVSKII
}

Abstract. In the paper the known results on couplings of symmetric operators $A_{j}, j \in\{1,2\}$, in the sense of A.V. Shtraus are extended to the case of operators $A_{j}$ with arbitrary (possibly unequal and infinite) deficiency indices. In particular, we generalize to this case the coupling method based on the theory of boundary triplets for symmetric operators. This enables us to obtain the abstract Titchmarsh formula, which gives the representation of the Weyl function of the coupling in terms of Weyl functions of boundary triplets for $A_{1}^{*}$ and $A_{2}^{*}$. In applications to differential operators on $\mathbb{R}$ this formula turns into the classical Titchmarsh formula, which gives a representation of the characteristic matrix $\Omega(\cdot)$ in terms of Titchmarsh-Weyl functions on semiaxes $\mathbb{R}_{+}$and $\mathbb{R}_{-}$. Moreover, by using the coupling method we parameterize all Naimark exit space extensions $\widetilde{A}=\widetilde{A}^{*}$ of the second kind of a densely defined symmetric operator $A$ with finite possibly unequal deficiency indices.

Mathematics subject classification (2010): 34B20 47A06, 47A20,47A48, 47A56, 47B25.

Keywords and phrases: Symmetric relation (operator), boundary triplet, Weyl function, coupling of symmetric relations, Titchmarsh formula, exit space extension, generalized resolvent.

\section{REFERENCES}

[1] N. I. AkHIEZer, I. M.Glazman, Theory of linear operators in Hilbert space, Vol. I and II, Pitman, Boston-London-Melbourne, 1981.

[2] Yu. M. ArlinskiI, V. A. Derkach And E. R. TSeKanovskit, On unitary equivalent quasiHermitian extensions of Hermitian operators, Mat. Fiz. 29 (1981), 72-77 (in Russian).

[3] F.V. ATKInson, Discrete and continuous boundary problems, Academic Press, New York, 1963.

[4] J. Behrndt, V. Derkach, F. Gesztesy, M. Mitrea, Coupling of symmetric operators and the third Green identity, Bull. Math. Sci. 8 (2018), 49-80.

[5] J. Behrndt, S. HASSi, H. DE SNOo, R. Wiestma, Square-integrable solutions and Weyl functions for singular canonical systems, Math. Nachr. 284 (2011), no 11-12, 1334-1383.

[6] J. Behrndt, M. LANGer, Boundary value problems for elliptic partial differential operators on bounded domains, J. Funct. Anal. 243 (2007), 536-565.

[7] M.S. BRODSKII, Unitary operator colligations and their chracteristic functions, Russian Mathematical Surveys 33(1978), no. 4, 159-191.

[8] V. M. BRUK, Extensions of symmetric relations, Math. Notes 22 (1977), no.6, 953-958.

[9] V.M. BRUK, Linear relations in a space of vector functions, Math. Notes 24 (1978), no 4, 767-773.

[10] A. DiJKsma AND H. LANGeR, Compressions of self-adjoint extensions of a symmetric operator and M.G. Krein's resolvent formula, Integr. Equ. Oper. Theory 90:41 (2018).

[11] V.A. Derkach, S. Hassi, M.M. Malamud, and H.S.V. De Snoo, Generalized resolvents of symmetric operators and admissibility, Methods of Functional Analysis and Topology 6 (2000), no. 3, $24-55$.

[12] V. A. Derkach, S. Hassi, M. Malamud, And H. S.V. De Snoo, Boundary Relations and their Weyl families, Trans. Amer. Math. Soc. 358 (2006), no. 12, 5351-5400.

[13] V.A. Derkach, S. Hassi, M.M. Malamud, and H.S.V. De Snoo, Boundary relations and generalized resolvents of symmetric operators, Russian J. Math. Ph. 16 (2009), no. 1, 17-60. 
[14] V.A. Derkach And M.M. Malamud, Generalized resolvents and the boundary value problems for Hermitian operators with gaps, J.Funct. Anal. 95 (1991),1-95.

[15] V.A. DeRKACH AND M.M. Malamud, The extension theory of Hermitian operators and the moment problem, J. Math. Sciences 73 (1995), no. 2, 141-242.

[16] V. A. DeRKACH AND M. M. Malamud, Extension theory of symmetric operators and boundary value problems, in Proceedings of Institute of Mathematics NAS of Ukraine, V.104, Institute of Mathematics NAS of Ukraine, Kyiv, 2017, 573 p.

[17] A. Dijksma, H. LANGer, H.S.V. DE SnOo, Eigenvalues and pole functions of Hamiltonian systems with eigenvalue depending boundary conditions, Math. Nachr. 161 (1993), 107-153.

[18] I. Gohberg, M.G. KReIn, Theory and applications of Volterra operators in Hilbert space, Transl. Math. Monographs, 24, Amer. Math. Soc., Providence, R.I., 1970.

[19] V.I. GORBACHUK AND M.L. GORBACHUK, Boundary problems for differential-operator equations, Kluver Acad. Publ., Dordrecht-Boston-London, 1991. (Russian edition: Naukova Dumka, Kiev, 1984).

[20] D.B. Hinton, A. SCHneIder, On the Titchmarsh-Weyl coefficients for singular S-Hermitian systems I, Math. Nachr. 163 (1993), 323-342.

[21] V.I. KhrabUSTOVSKy, On the characteristic operators and projections and on the solutions of Weyl type of dissipative and accumulative operator systems. 3. Separated boundary conditions, J. Math. Phis. Anal. Geom., 2 (2006), no 4, 449-473.

[22] A. N. KochubeI, On extensions and characteristic functions of symmetric operators, Izv. Akad. Nauk. Arm. SSR 15 (1980), 219-232. (In Russian); English translation: Soviet J. Contemporary Math. Anal. 15 (1980).

[23] V.I. KOGAN AND F.S. RofE-BEKETOV, On square-integrable solutions of symmetric systems of differential equations of arbitrary order, Proc. Roy. Soc. Edinburgh Sect. A 74, (1974/75), 5-40.

[24] A.M. KRALL, $M(\lambda)$-theory for singular Hamiltonian systems with one singular point, SIAM J. Math. Anal. 20 (1989), no 3, 664-700.

[25] A.M. KRALL, $M(\lambda)$-theory for singular Hamiltonian systems with two singular points, SIAM J. Math. Anal. 20 (1989), no 3, 701-715.

[26] M.G. KREIN AND H. LANGER, On defect subspaces and generalized resolvents of a Hermitian operator in the space $\Pi_{\kappa}$, Funct. Anal. Appl. 5 (1971/1972), 136-146, 217-228.

[27] S. KUZHel AND L. NIZHNiK, Phillips symmetric operators and their extensions, arXiv:1801.04915v2 [math.FA] 21 Jan 2018.

[28] H. LANGER AND B. TEXTORIOUs, On generalized resolvents and $Q$-functions of symmetric linear relations (subspaces) in Hilbert space, Pacif. J. Math. 72(1977), no. 1, 135-165.

[29] M. LesCH AND M.M. MALAMUD, On the deficiency indices and self-adjointness of symmetric Hamiltonian systems, J.Differential Equations 189 (2003), 556-615.

[30] M. M. MALAmud, On the formula of generalized resolvents of a nondensely defined Hermitian operator, Ukr. Math. Zh. 44(1992), no. 12, 1658-1688.

[31] M. M. Malamud And V. I. MogilevskiI, Generalized resolvents of isometric operators, Mat. Notes 73(2003), no.3-4, 429-435.

[32] M. M. Malamud And V. I. Mogilevskit, Resolvent matrices and spectral functions of an isometric operator, Dokl. Akad. Nauk 395(2004), no. 1, 11-17 [Dokl. Math. 69(2004), 158-163.].

[33] V.I. Mogilevs KiI, Nevanlinna type families of linear relations and the dilation theorem, Methods Funct. Anal. Topology 12 (2006), no. 1, 38-56.

[34] V.I. Mogilevs KiI, Boundary triplets and Krein type resolvent formula for symmetric operators with unequal defect numbers, Methods Funct. Anal. Topology 12(2006), no. 3, 258-280.

[35] V. MogilevsKiI, On characteristic matrices and eigenfunction expansions of two singular point symmetric systems, Math. Nachr 288(2015), no. 2-3, 249-280.

[36] V.I. Mogilevskit, On exit space extensions of symmetric operators with applications to first order symmetric systems, Methods Funct. Anal. Topology 19 (2013), no. 3, 268-292.

[37] V. MogiLeVs KII, On eigenfunction expansions of first-order symmetric systems and ordinary differential operators of an odd order, Integr. Equ. Oper. Theory 82 (2015), 301-337.

[38] B. Sz.-Nagy, C. FoiAs, Harmonic Analysis of Operators in Hilbert Space, Paris and Akad.Kiado, Budapest, 1967.

[39] O. Post, Boundary pairs associated with quadratic forms, Math. Nachr. 289 (2016), 1052-1099. 
[40] A.V. S̆TRAUS, On generalized resolvents and spectral functions of differential operators of an even order, Izv. Akad. Nauk. SSSR, Ser.Mat., 21 (1957), 785-808.

[41] A. V. Shtraus, Characteristic functions of linear operators, Izv. Akad. Nauk SSSR. Ser. Mat. 24 (1960),no.1, 43-74. (Russian); English translation: Amer. Mafh. Soc. Transl, 2(1964), 40, 1-37.

[42] A.V. Shtraus, On selfadjoint operators in the orthogonal sum of Hilbert spaces, Dokl. Akad. Nauk SSSR, 144 (1962), no.3, 512-515.

[43] A. V. ShtRaus, On extensions and characteristic functions of symmetric operators, Izv. Akad. Nauk SSSR. Ser. Mat. 32 (1968),no.1 186-207. (Russian); English translation: Mathematics of the USSRIzvestiya, 2 (1968), no. 1, 181-203.

[44] E.C. Titchmarsh, Eigenfunction expansions associated with second-order differential equations, Part 1, Clarendon Press, Oxford, 1962. 